\section{A CASE OF DILATATION OF THE STOMACH TREATED BY AN OPERATION BELIEVED TO BE NEW.}

By WILIIAM EWART, M.D. CANTAB., F.R.C.P. LOND., PHY'SICIAN TO ST. GEORGE'S HOSPITAL;

\section{AND}

WILLIAM H. BENNETT, F.R.C.S. EYG., SURGEON TO ST. GEORGE"S HOSPITAL.

THE time which has elapsed since the date of the operation adopted in the following case is not yet sufficient to justify any positive conclusion as to the permanence of the benefit derived by the patient. We, however, feel justified in recording the case as the results obtained up to the present time (March, 1896) are so good that we believe the treatment to be worthy of consideration and trial at the hands of others in appropriate cases of gastric dilatation.

A man aged thirty-four years, a native of South Australia, was admitted into St. George's Hospital under the care of Dr. Ewart on Nov. 10th, 1895, with the following history, the particulars of which were taken by Mr. E. L. Hunt. He had always been in perfect health until about eight fyears previously, when he was laid up in Adelaide with typhoid fever, from which he recovered in nine weeks. Almost immediately afterwards he had an attack of rheumatic fever, which incapacitated him for four months or thereabouts. Soon after recovering from the rheumatic fever he began to suffer from "spasms" in the stomach, especially after food. Since that time he had been a constant sufferer from painful dyspepsia, which at times had been so bad as to cause him to take to his bed. For four or five years he had suffered from obstinate constipation and great flatulent distension with serere pain almost continually. In the summer of 1894 these symptoms became so severe that all attempts at following his occupation (that of a carpenter) had to be relinquished. Having been treated for several years in New York and the United States for relief of his symptoms without effect he came to London and was admitted to another hospital as "a case of dilated stomach," which was treated by daily washing 3 , de. He remained in the hospital for six weeks, and since learing it he had washed out his stomach assiduously every day with very partial relief. Before leaving that hospital the question of operation was discussed, but nothing further was done. For three or four years there had been progressive loss of flesh. On admission into St. George's Hospital the patient was found to be greatly emaciated, the features were sharp and the cheeks hollow, there was good energy of voice and caanner, and the appearance generally was rather that of imperfect nutrition than of disease. The chief symptoms complained of were an extreme constant dragging pain in the right abdominal regions especially when standing; pain at the epigastrium after food, which sometimes extended to the left cardiac area; habitual obstinate constipation; and attacks of anorexia with persistent and almost intolerable flatulence. On examination the organs were found to be normal with the exception of the stomach, which was greatly enlarged (see Fig. 3), and gave a gross splashing sound on percussion which the patient could also himself elicit br shaking his body. For a few days he was kept in bed and treated for the dyspeptic symptoms with complete relief to the dyspepsia and also to the constipation; on being allowed to get up the symptoms, however, returned as before. The patient's misery was so great that he begged urgently for some relief by operation if such were possible, and stated that ho was prepared to run any risk as his present condition was unbearable, and that the washing 5 and stomach "toilet" which he had for a time practised were not only almost impracticable for him to carry out but also gave only partial relief. A consultation was therefore held between Dr. Ewart and Mr. Bennett, at which it was decided to carry out a method which it was thought would afford the necessary resuit. On Nov. 26th (after a consultation with his surgical colleagues) Mr. Bennett performed the following operation. The abdomen having been opened by a median incision about five inches long, cacmencing above at a point an inch below the ensiform appsndix, the stomach was thoroughly examined by the hand introduced trroigh the wound. No adhesions were detected and the pylorus was normal. The viscus was then drawn out of the abdomen and laid upon the parietes, where it presented a large balloonlike bag distended with gas. A fold on the anterior aspect of the stomach was then turned in by the fingers of the assistant (Dr. Nachbar, the house surgeon). The length of this fold, which followed in direction the long axis of the stomach, was about twelve inches, its greatest depth being three inches. The inverted portion was, therefore, about six inches in width at its widest part and twelve inches long (Fig. 1). Whilst this fold was kept

FIG. 1.

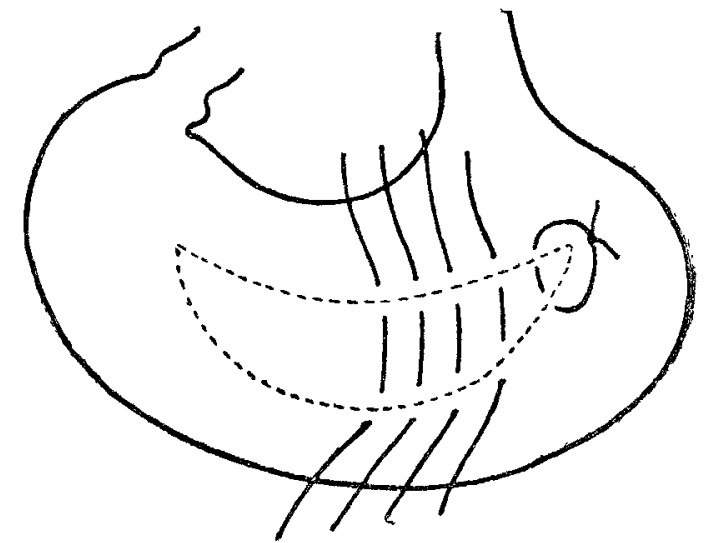

Diagram showing the arrangement of sutures. The dotten lines indicate the extent and shape of the inverted area of the stomach wall.

involuted by the assistant's fingers the peritoneal surfaces on the opposite sides of its base were brought together by numerous sutures made to transfix the peritoneal and muscular coats in the manner indicated in Figs. 1 and 4 . the fingers of the assistant being gradually withdrawn from the fold before the tightening up of the finas stitches. After the stitches had all been fastened off the size of the stomach was greatly lessened, the shape of the viscus then being that shown in Fig. 2. Mr. Bennet

FIG. 2.

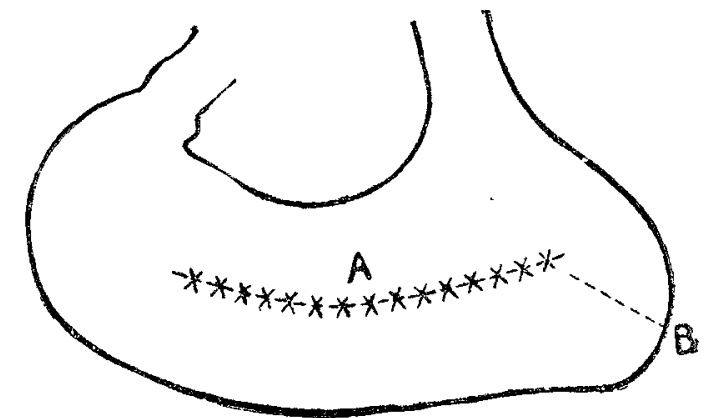

Diagram showing the alteration in the shape of the stomach effected by the operation. A: the sutured edges of the base of the secondary involution for obviating the dependent tendency of the great end of the stomach.

had intended to make a secondary small involution at the great end of the stomach (Fig. 2, B) to prevent the otherwise inevitable dependent tendency of that part, but as the patient was in a very unsatisfactory condition generally it was thought wiser to be content with what had already been done. The altered viscus was then returned into the abdomen, the wound in the parietes being closed by fish-gut sutures in the usual way.

Progress of the case.-The patient romited once soon after the operation. As soon as he was fully conscious he was frequently given small quantities of water, as hot as could be tolerated, by the mouth. At 6.30 P.M. (the operation having been completed at 3.15) Mr. Bennett, as the collaper was still marked, himself saw administered an egg beaten up with an ounce of brandy. This having been retained witls comfort further nourishment by the mouth in the shape of brandy and meat essence was given throughout the night when the patient, who slept a good deal, was awake, thirst when complained of being assuaged with hot water in small quantities frequently giren. On Nov. 27 th the patient, having had a very fair night, was a little sick during the morning, but without straining. He took nourishment wellriz., two eggs, extra strong beef-tea, meat essence, and two pints of milk. (This dietary was continned until 
Dec. 1st, when chicken and custard were added; milkpudding was also added on the 8th, greens on the 18th, and he was ordered a mutton chop on Jan. 18th, 1896.) On Nov. 29th the wound was inspected as the dressings were uncomfortable. It was quite healthy in appearance. There had been no more sickness and hardly any pain except a slight dragging pain on deep breathing. The pulse and temperature were normal. On Dec. 1st there had been much improvement. Food was taken without any difficulty, there was no feeling of sickness or pain, the bowels were open, the pulse was 72 , full and strong, and the temperature, which was slightly sub-normal before the operation, had risen to about normal with occasionally a few tenths of a degree of pyrexia in the evening. On the 6th all the stitches were removed from the abdominal wound, which had quite healed. There was no discomfort of any kind and the patient felt very well and was placed on a more substantial diet. The bowels were, however, a little distended. On the 8th he got up for two and a half hours. Some pain was complained of in the left thigh along the saphenous vein, which was varicose. There was no rise of temperature. On the 10th there was marked tenderness along the saphenous vein of the left thigh; there was no sign of phlebitis. (Upon inquiry it appears that the patient had an operation performed for varicose veins in New York, the veins " being pinned"; since that operation he has been subject at times to great pain in the veins.) All stomach trouble had disappeared; there was neither pain nor discomfort after food. The pain in the limb, which was kept at rest, was disappearing. On the 18th all pain in the limb had subsided and the patient Eelt very well. On the 23rd he complained of a slight sensation of dragging at the abdominal scar on standing up; otherwise he was quite well. On the 27th the patient was allowed to remain up permanently; but he still had slight dragging pain. Up till Jan. 14th there was practically nothing further to note. The patient had occasionally complained of some very slight discomfort after meals and also said that he had noticed a suggestion of the old splash; but on examination this could not be elicited either by gentle tapping of the anterior abdominal wall as previously or even by moderate succussion. The size of the stomach was carefully determined by auscultatory percussion on Dec. 16th, and the tympanitic area was found to be considerably reduced (Fig, 3). A further

FIG. 3.

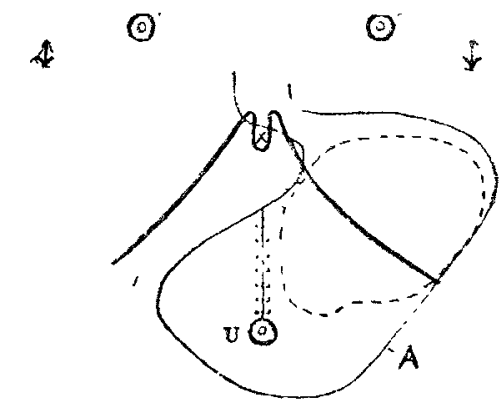

Tracing showing the tympanitic areas found by auscultatory percussion before and after the operation (from a photograph taken by Mr. Powell). A : tracing obtained prior to th operation. The dotted line shows the reduced area of resonance after the operation. $\mathrm{U}$ : umbilicus, above which is the line of incision. $x$ : the ensiform appendix. The right and left nipples are also shown. and the arrows indicate the position of the mid-axillary line.

tracing taken a fortnight later gave practically the same result. Since the operation no medicine was administered, with the exception of some acid glycerine of pepsine, an occasional aperient pill, and an occasional carminative dose. For several weeks past his weight had remained stationary; he had regained $2 \mathrm{lb}$. since the original loss mentioned above. $\mathrm{He}$ was still somewhat thin and hollow-cheeked, but was greatly improved in aspect, and his general nutrition was much better than before operation. The patient left the hospital at the end of January, 1896, greatly improved in all respects. The stomach resonance remained the same as in the diagram (Fig, 3); there was no constipation and no dyspeptic symptoms were occurring; the only discomfort complained of was an occasional slight dragging about the abdominal scar, but this was gradually diminishing and was entirely relieved by the use of a light abdominal belt with which the patient had been provided.

Remarks by Dr. EWART. - The uselessness of internal remedies and of the stomach tube having been amply proved before the patient's admission into St. George's Hospital under my care, attention was at once given to the framing of a diagnosis sufficiently accurate, if possible, to be of some use in the selection of the mode of surgical relief. Was this a case of pyloric obstruction, and, if so, was it cancerous in nature or merely fibrous, or was it an instance of simple dilatation, and, if so, had the changes progressed to the stage of atrophy of the mucous membrane and fibrous gastrectasia? These were the chief alternatives which presented themselves for consideration. Although it was difficult to exclude absolutely the possibility of carcinoma, which was suggested by some of the symptoms and by the patient's emaciation, still the positive signs of cancer were absent. There had never been any hæmatemesis, and no tumour could be felt in the epigastrium. A careful review of the symptoms negatived pyloric stricture or even any considerable narrowing of the pyloric orifice. There had been no vomiting either before the systematic use of the stomach tube or since this was discontinued. The recurring gastric attacks had been such as might arise from undue stagnation and accumulation of the contents of a dilated stomach, but the pyloric passage remained clear, and with the help of posture the food had invariably found its way down the intestine. The view that the condition was one of dilatation only, received strong support from the history of its origin. Immediately after a severe attack of typhoid fever the patient suffered from acute rheumatism. Wach of these two diseases is an adequate and recognised cause of gastric dilatation; but their sequence in the order stated, in which the recurring inflation incidental to the administration of the bicarbonates and to a fever diet is long kept up in a stomach previously starved and weakened, would of all combinations be the most likely to lead to the result, quite independently of any pyloric affection. Lastly, there was good reason to regard the stomach as free from much atrophy of the mucous membrane. Digestion was at times much disturbed, but between the severe gastric seizures there were intervals of relatively normal function, and the results of test meals administered after preliminary washing out of the stomach were satisfactory. The diagnosis arrived at was, therefore, simple atonic dilatation without pyloric obstruction. Believing that the gastric mucous membrane was in a fairly healthy condition and the pylorus free from disease I should have been disinclined to recommend any procedure (such, for instance, as gastro-jejunostomy) which would interfere with the integrity of the stomach. I had long felt that in cases of this kind the surgical indication was to rectify the faulty mechanical conditions and to relieve in some way the tendency to sacculation. Mr. Bennett had formerly suggested in discussing the matter an operation by which the indication might be fulfilled. The present case struck me as being singularly well adapted for the successful employment of the method in question; I therefore ventured to strongly advocate its performance. The results have so far entirely justified the treatment, as may be seen from the account of the case.

Remarks by Mr. BenNeTT. - It cannot be denied that dilatation of the stomach unassociated with pyloric obstruction may be productive of the greatest misery to the patient and may even in some cases lead to a condition bordering upon starvation. In the majority of instances, it is true, life may be continued with varying degrees of pain or discomfort, provided that the stomach be frequently washed out and the necessary restrictions in diet and in the manner of living be rigidly observed. At the same time it must be admitted that the frequent washings and details generally of the stomach "toilet" in these cases are at the best distressful and often revolting to susceptible subjects, by whom the treatment is carried out reluctantly, and as often as not solely because of the fear of the pain and romiting which may follow upon its omission. In certain classes it is obvious that the treatment referred to is practically impossible, and even with the wealthy and otherwise well circumstanced the treatment is often almost intolerable and has been the determining factor in the serious contemplation of self-destruction. Certain cases have from time to time been recorded in which the separation of adhesions about the stomach after abdominal section has been followed by complete relief of the symptoms complained of in dilatation, and in which a shrinking in the abnormally large viscus has come about. In cases in which no adhesions exist it is clear that nothing short of some actual diminution in the size of the stomach 
by operative measures can be relied upon to give practical relief. The obvious proceeding which first suggests itself is the removal of a portion of the whole thickness of the stomach of a sufficient size to produce the desired decrease in capacity, but this operation is unduly severe and could hardly be free from certain risks when the thickness and vascularity of the stomach walls are considered on the one hand, and the very extensive area which would require excision on the other hand in these cases of very large stomachs. After consideration, therefore, I came to the conclusion that the necessary diminution in the stomach capacity could be brought about by doubling in a portion of the walls upon the lines indicated in the description of the case now recorded. This operation is clearly much less severe than any excision of a portion of the stomach could be, and, moreover, although the stomach is itself decreased in size the whole of the mucous membrane is left untouched, which must be distinctly advantageous for purposes of digestion. After a discussion of the subject with my colleague, Dr. Ewart, it was determined to test the treatment in the next case of a sufficiently severe nature which came under observation, as the risk appeared to hardly exceed that of mere abdominal section, which in these times cannot be considered as serious. The only theoretical objection which could be urged against the proceeding appeared to be a possibility that the large infolded flap of mucous membrane might during the contraction of the stomach form an obstructive mass in the pyloric region and so prevent the proper outflow of the contents of the stomach. This objection, however, has no practical value if in the making of the fold care be taken first to avoid encroaching too closely upon the pylorus, and, secondly, to see that the fold follows accurately the long axis of the stomach. As is often the case in an operation performed for the first time on the living subject, there are certain technical defects in the proceeding adopted in the present case which I shall in the future endeavour to avoid, although they in no sense appear to have invalidated the satisfactory result of the treatment up till now in this patient. The defects to which I refer are two in number; the first is the unduly dependent position in which the great end of the stomach remains, which is obviously undesirable, but can easily be rectified in another case either by continuing the main involution along to the extreme cardiac edge of the viscus, or, as I had intended to do in the present case had circumstances allowed, by making a small secondary involution at the situation marked $\mathrm{B}$ in Fig. 2. The second defect is one which may or may not be of importance, but I conceive that it may possibly interfere with the permanence of the benefit derived; it can, however, be easily obviated in the future and in no way affects the principle of the operation. It will be seen by reference to A, Fig. 4, that the line of apposition

FIG. 4.

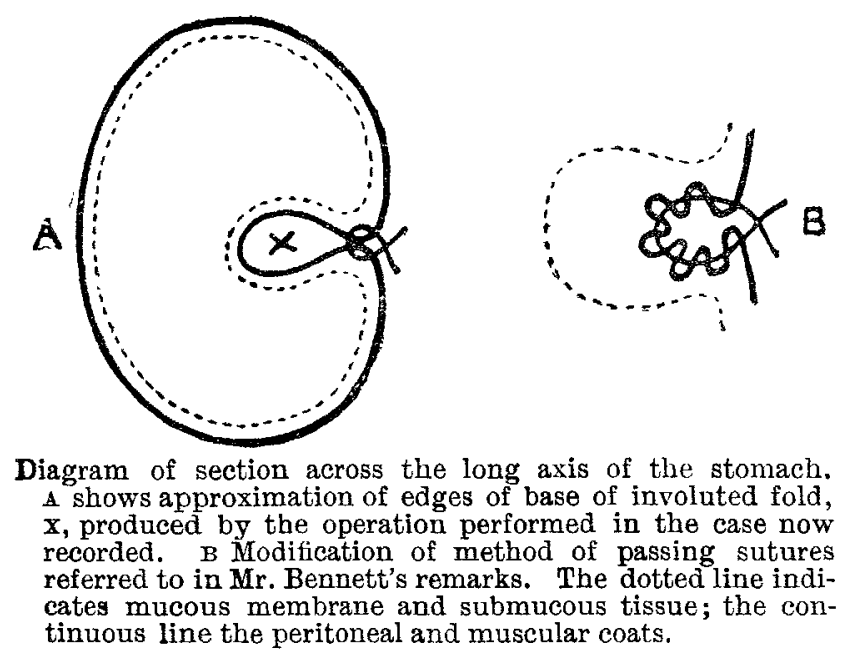

of the opposite peritoneal edges produced by sutures introduced in the manner described is rather narrow; it is therefore conceivable that the line of union as it is so thin may in course of time stretch and so lead to a recurrence of the dilatation. The future history of the case will, of course, settle the point definitely. In the meantime, should another case present itself which is appropriate for operation I shall avoid this possible defect by passing the sutures by threading in and out across the area intended to be turned in, after the manner shown in Fig. 5, no suture being fastened until all have been introduced. The tightening up of stitches each arranges in this purse-string-like fashion will produce an extensive puckering up of the included peritoneal surface in the way indicated in Fig. 4, B, the resulting union being so complicated and extensive that stretching must be practically out of the question. This modification of the operation, although it appears at first sight to add complication to the plan, in reality makes it more easy, since no involution by the fingers of an assistant is necessary, the stitches being merely threaded in and out across the portion of the stomach it is proposed to inver's. The extent of the fold included in the stitches can also in this way be much more accurately gauged than when the fold is turned in by the fingers, as its extent must then be in a certain degree doubtful. As will be gathered from the notes of the case now recorded the relief afforded by the operation was remarkable, and, so far as can be judged from the present condition of the patient, there seems to be no reason why it should not be permanent in spite of the apparent possible defects in the technical results of the pro. ceeding to which I have alluded. A noteworthy point in the case is the singular tolerance shown by the stomach for nourishment given by the mouth almost immediately after the operation. A considerable experience of operation upon the stomach confirms me in the conviction, which 1

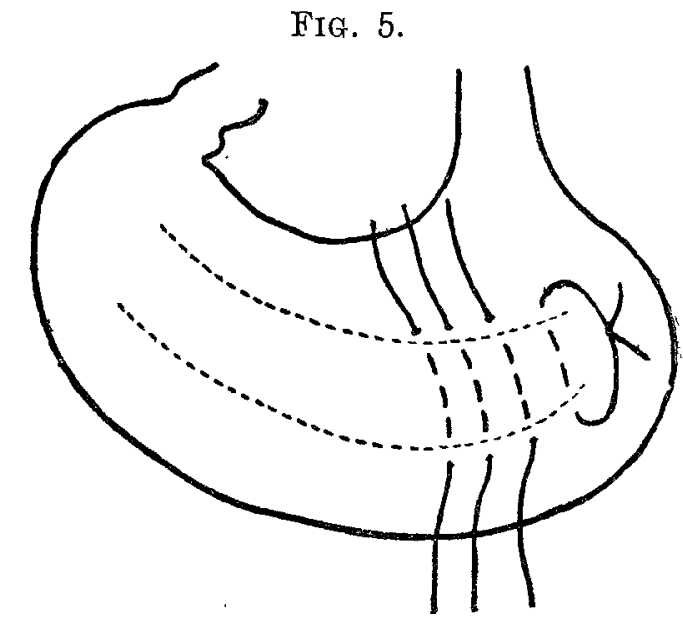

Diagram showing modified plan of introducing sutures for the purpose of obtaining larger area of adhesion of the sides of the infolded flap. The result of this modification is seen in Fig. 4, $B$.

have frequently expressed, that in cases of operation involving this viscus nourishment by the natural passage cannot be given too soon after the patient has recovered sufficiently from the anæsthetic to be able to swallow. It is further, I think, undeniable that the surest means for counteracting the shock from which these patients succumb when they die soon after operation, is to give at once, in small quantities at a time but freely, water as hot as it can be borne, and then as soon as the stomach has learnt to tolerate water only to begin the administration of liquid or jelly nourishment by the mouth. This plan I have myself always followed, and it is mainly to that practice that I attribute the fact that every patient upon whom I have up to the present time performed operations involving the stomach-e.g., gastro-jejunostomy and suture of perforating gastric ulcer-has recovered, although in two of the cases the patients were at the end of the operation apparently in an utterly hopeless condition. The results of the case described in this communication are in my opinion so good that I shall certainly perform the operation in any other appropriate case which may fall into my hands. The principle of the treatment is, I submit, sound, and the risk entailed in carrying it out cannot with ordinary care be great. It must not be for one moment inferred that the operation is suggested as a routine treat ment for gastric dilatation; but for extreme cases in which treatment by the stomach "toilet" is either impracticable, intolerable, or ineffective the radical method adopted in this case may, I believe, be considered a rational proceeding, and may be undertaken by any competent surgeon without sub. jecting the patient to risks which are out of proportion to the amount of benefit likely to be derived from the operation.

NoTE.- After leaving the hospital the patient resumed his work as a carpenter. Recently (June 17th) he has reported himself complaining of some dyspeptic symptoms. There is a modified stomach splash and the area of resonance is rather larger than that shown in Fig. 3. The sequel of the case will be published later. 